\title{
On the Probability for Sub-Halo Detection through Quasar Image Splitting
}

\author{
T. Riehm ${ }^{1}$, E. Zackrisson ${ }^{1,2,3}$, K. Wiik ${ }^{2}$ and O. Möller ${ }^{4}$ \\ ${ }^{1}$ Stockholm Observatory, AlbaNova University Center, 10691 Stockholm, Sweden \\ ${ }^{2}$ Tuorla Observatory, University of Turku, Vislntie 20, FI-21500 Piikki, Finland \\ ${ }^{3}$ Department of Astronomy and Space Physics, Box 515, 75120 Uppsala, Sweden \\ ${ }^{4}$ Max Planck Institut fr Astrophysik, D-85741 Garching, Germany
}

\begin{abstract}
Dark galaxies may in principle be detectable through strong-lensing image splitting of quasars on small angular scales (milliarcseconds or below). Here, we estimate the overall probabilities for such detections under the assumption that the quasars can be treated as point sources. Due to the very low probabilities derived, we conclude that it is currently not feasable to use this strategy to put the CDM predictions for the dark galaxy population to the test.
\end{abstract}

Keywords. dark matter - cosmology: observations - galaxies: halos - quasars: general

\section{CDM subhalos}

In the CDM scenario (as well as in several slight modifications thereof), dark matter halos are assembled hierarchically from smaller subunits. A long-standing problem with this picture is that the number of subhalos predicted by CDM simulations at any given time is orders of magnitudes higher than the known number of satellite galaxies in the the vicinity of the Milky Way. There are several possible ways out of this dilemma: either the CDM scenario is incorrect, the simulation predictions are wrong, or the majority of these subhalos must somehow have evaded detection. The latter alternative is quite viable, provided that the baryonic content of these subhalos has either been lost or prevented from forming stars. If such "dark galaxies" do indeed exist, gravitational lensing may offer one of the most promising ways to detect them.

\section{Gravitational millilensing by subhalos}

One tell-tale signature of dark matter subhalos in the $10^{6}-10^{10} \mathrm{M}_{\odot}$ range would be gravitational millilensing, i.e. image splitting with a characteristic separation of milliarcseconds (e.g. Yonehara et al. 2003). Based on a null detection of millilensing in a sample of 300 quasars observed with the VLBI, Wilkinson et al. (2001) demonstrated that the vast majority of quasars do not show any signs of millilensing, and were able to impose upper limits of $\Omega ; 0.01$ on the cosmological density of dark matter objects in this mass range. Unfortunately, this constraint is still insufficient to rule out the subhalos predicted by CDM, since their optical depth is expected to be at least one order of magnitude lower. To put the CDM subhalo predictions to the test, Yonehara et al. (2003) instead suggested that one should target quasars which are already known to be gravitationally lensed on arcsecond scales, as one can then be sure that there is a massive halo well-aligned with the line of sight, which substantially increases the probability for subhalo millilensing. Indeed, the magnification associated with millilensing has long been suspected to be the cause of the flux ratio anomalies seen in such systems (e.g. Mao \& Schneider 1998; 
Kochanek \& Dalel 2004). Subhalo millilensing has also been advocated as an explanation for strange bending angles of radio jets (Metcalf 2002) and image positions which smooth halo models seem unable to account for (Biggs et al. 2004).

\section{Prospects for direct detection of dark matter subhalos through gravitational lensing}

Under the assumption that the lenses do not overlap along the sightline, the optical depth $\tau$ represents the fraction of the sky that is covered by regions in which a point source will be lensed. In the limit of small $\tau$, the optical depth can therefore directly be used as an estimate of the lensing probability. Assuming dark matter halos to be Singular Isothermal Spheres (SIS) and using the subhalo mass function and number density profile given in Gao et al. (2004), we derived estimates for the optical depth $\tau$ as a function of impact parameter to the center of the host halo. Even when allowing for a variety of host halo masses and redshift combinations, $\tau$ does not exceed a value of $10^{-3}$. This is contrary to previous estimates (e.g. Yonehara et al. 2003) indicating optical depths several magnitudes higher. In order to be able to use this method to put the CDM subhalo predictions to a test, a survey of more than $10^{3}$ objects would be required, in contrast to the $\sim 10^{2}$ multiply-imaged quasars presently known. Moreover, since it would be highly time consuming to observe on the order of $10^{3}$ objects at milliarcsecond resolution using existing facilities, a search for CDM subhalos is currently not feasable. Furthermore, using SIS cross sections results only in an upper limit for the optical depth. Considering more realistic, less concentrated halo profiles, e.g. a Navarro-Frenk-White(Navarro et al. 1996) or Moore-profile (Moore et al. 1999), would reduce the overall probabilities for detecting subhalos through this technique even more (Zackrisson et al. 2007). These results are valid for point sources. Whether extended sources (as in the case of VLBI observations of quasars) can boost the probability for subhalo millilensing is something that we are currently looking into (Riehm et al., in preparation).

\section{Acknowledgements}

EZ acknowledges research grants from the Swedish Research Council, the Academy of Finland and the Swedish Academy of Sciences.

\section{References}

Biggs, A. D., Browne, I. W. A., Jackson, N. J., York, T., Norbury, M. A., McKean, J. P. \& Phillips, P. M. 2004, MNRAS 350, 949

Gao, L., White, S. D. M., Jenkins, A., Stoehr, F. \& Springel, V. 2004, MNRAS 355, 819

Kochanek, C. S. \& Dalal, N. 2004, ApJ 610, 69

Mao, S. \& Schneider, P. 1998, MNRAS 295, 587

Metcalf, R. B. 2004, ApJ 580, 696

Moore, B., Quinn, T., Governato, F., Stadel, J. \& Lake, G. 1999, MNRAS 310, 1147

Navarro, J. F., Frenk, C. S. \& White, S. D. M. 1996, ApJ 462, 563

Wilkinson, P. N., et al. 2001, Phys. Rev. Lett. 86, 584

Yonehara, A., Umemura, M. \& Susa, H. 2003, PASJ 55, 1059

Zackrisson, E., Riehm, T., Mller, O., Wiik, K. \& Nurmi, P. 2007, submitted to ApJ 